\title{
Implications of Solar Magnetograms for the Drifts of Cosmic Rays
}

\author{
Horst Fichtner $^{a, b, *}$ and Andreas Kopp ${ }^{a, c}$ \\ ${ }^{a}$ Institut für Theoretische Physik IV, Ruhr-Universität Bochum, \\ Universitätsstrasse 150, 44780 Bochum, Germany \\ ${ }^{b}$ Research Department Plasmas with Complex Interactions, Ruhr-Universität Bochum, \\ Universitätsstrasse 150, 44780 Bochum, Germany \\ ${ }^{c}$ Centre for Space Research, North-West University, \\ 2520 Potchefstroom, South Africa \\ E-mail: hf@tp4.rub.de, ak@tp4.rub.de
}

\begin{abstract}
While gradient and curvature drifts are well-established elements of the propagation of cosmic rays in the heliospheric magnetic field, their perturbation by the Solar activity-induced largescale distortions of dipole-like field configurations even during Solar minima and by magnetic turbulence is an open problem. Various empirical or phenomenological approaches have been suggested to quantify these effects so that they can be straightforwardly incorporated in modulation models covering the 22-year periodicity (including the sign) of Solar activity. These approaches, however, either lack clear physics-based parametrizations (e.g., in terms of the tilt-angle of the heliospheric current sheet) or have been shown to be incompatible with measurements (like a dependence on the normalized turbulence level $\delta B / B$ ). We propose here a new approach to the treatment of drifts over an entire Solar cycle including maximum periods, which is based on Solar magnetograms. This not only provides a physics-based approach to the reduction of drifts during Solar activity maxima but also a treatment that is fully consistent with those MHD models of the Solar wind and the embedded heliospheric magnetic field that exploit Solar magnetograms as inner boundary conditions.
\end{abstract}

$37^{\text {th }}$ International Cosmic Ray Conference (ICRC 2021)

July 12th - 23rd, 2021

Online - Berlin, Germany

\footnotetext{
${ }^{*}$ Presenter
} 


\section{Introduction and motivation}

Even after many years of research and despite significant progress regarding the modeling of the transport of energetic charged particles in turbulent magnetic fields it is unclear whether a combination of current models of the kinetic transport of cosmic rays with magnetohydrodynamic (MHD) models of the turbulent Solar wind plasma turbulence can satisfactorily explain threedimensional (3D) multi-point spacecraft data, as, e.g., presented in [1].

In an attempt to help to clarify this issue, in a series of papers [2-4] we developed a numerical model suite to investigate the cosmic ray transport in realistic configurations starting from magnetograms provided by the Global Oscillation Network Group [GONG, 5] to construct the inner boundary conditions at the so-called heliobase [6] which we assume to be located at $0.1 \mathrm{AU}$. This configuration is propagated outward by means of the MHD code CRONOS [7] and taken as an input for the computation of the cosmic ray transport via Stochastic Differential Equations [8]. As a closer investigation [3] revealed, a proper treatment of particle drifts is essential. This applies already to the quiet conditions at Solar minimum and becomes even more critical at Solar maximum conditions when the configuration is much less regular. In order to fit numerical simulations to observational data, drifts have, in addition to a proper treatment, to be reduced when Solar activity is high [see, e.g. 9, 10, and references therein].

Here we briefly present a new method for the drift reduction, which is studied in greater detail in [4].

\section{Traditional models of drift reduction}

Particle drifts in the heliosphere, $\left\langle\vec{v}_{\mathrm{d}}\right\rangle$, are described by the expression [e.g. 11, and references therein]:

$$
\left\langle\vec{v}_{\mathrm{d}}\right\rangle=\nabla \times\left(\kappa_{\mathrm{A}} \frac{\vec{B}}{B}\right) .
$$

Here $\left\langle\vec{v}_{\mathrm{d}}\right\rangle$ denotes the pitch-angle averaged guiding center drift velocity and

$$
\kappa_{A}=q A \kappa_{\mathrm{A}, 0} \frac{P \beta}{3 B} \frac{\left(P / P_{0}\right)^{2}}{1+\left(P / P_{0}\right)^{2}} .
$$

is the modified drift coefficient, where $\vec{B}$ is the heliospheric magnetic field with $B=|\vec{B}|$. Further quantities are the ratio of the particle speed to the speed of light, $\beta$, the particle charge, $q$, and the rigidity, $P$ (with $P_{0}=(1 / \sqrt{2}) G V$ ). The factor $A$ is the sign of the Solar cycle and takes only the values \pm 1 . It determines the orientation (i.e. direction) and the form of the drift patterns [see 11, and references therein].

The drift reduction during periods of high Solar activity is usually performed by reducing the quantity $\kappa_{\mathrm{A}, 0}$ which is unity at full strength. Measures for Solar activity are the tilt-angle [12] or the level of magnetic field turbulence via the ratio of magnetic field fluctuations and the magnitude of the magnetic field [e.g., 13, and references therein]. While the latter does not show the periodicity of a Solar cycle [see 4], so that it cannot map the drift reduction over an entire Solar cycle, reductions via the tilt-angle are more or less heuristic models, but are able to reproduce observational data. We follow, thus, the ansatz via the tilt-angle, but try to put it on an improved physical basis. 

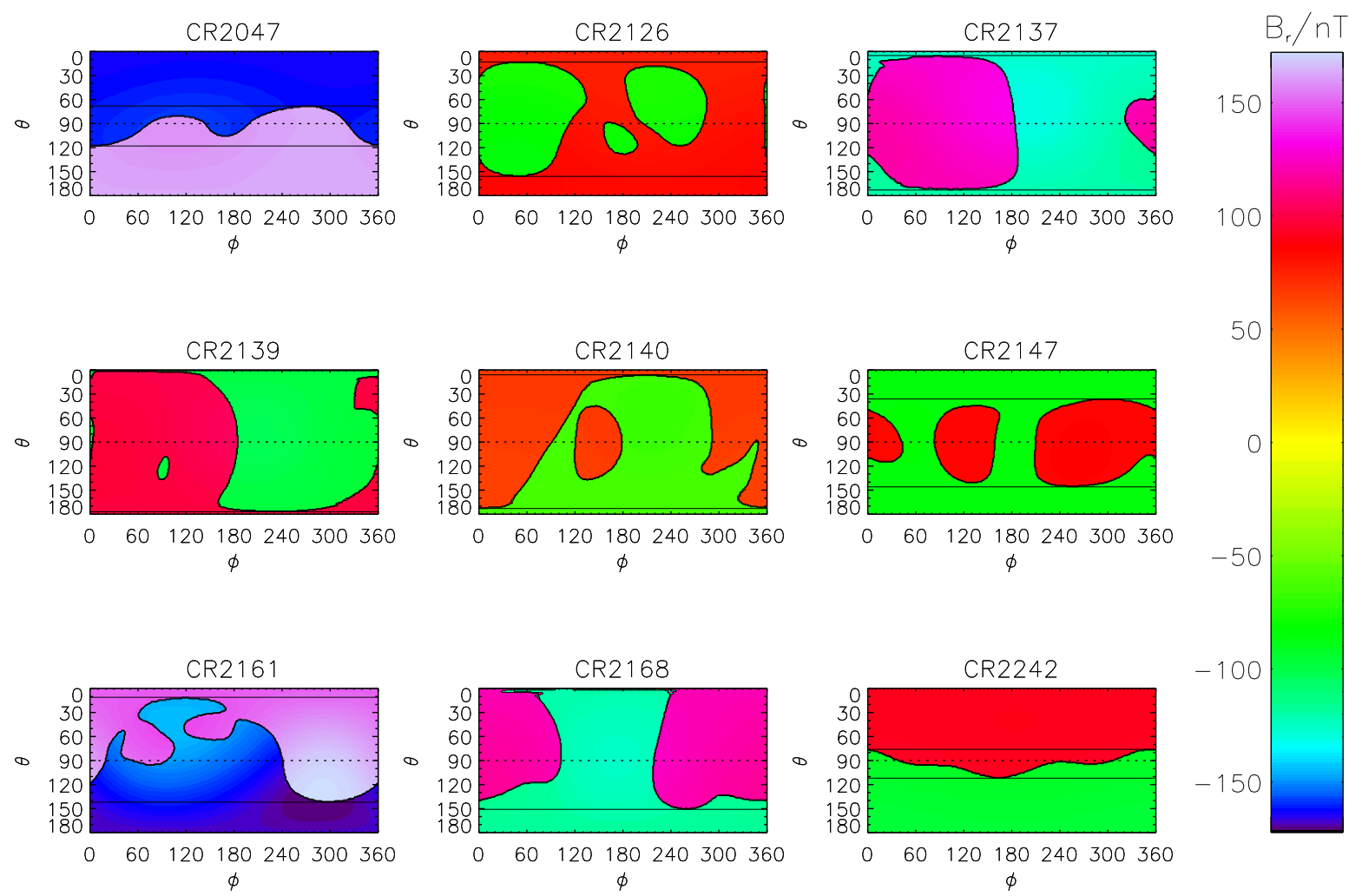

$-100$

$-150$

Figure 1: The radial magnetic field strength (with sign), $B_{\mathrm{r}}$ at the heliobase, which is located at $0.1 \mathrm{AU}$ for nine selected Carrington Rotations covering the time interval covered here.

The tilt-angle is defined as the maximum extent of the Heliospheric Current Sheet (HCS) from the equator in northern $\left(\alpha_{\mathrm{N}}\right)$ and southern $\left(\alpha_{\mathrm{S}}\right)$ directions. These can be obtained from the Wilcox Solar Observatory (WSO) [see 14, and the respective website for the full data from 1976 until today: http://wso.stanford.edu/]. Below, we will define tilt-angles in a somewhat different way.

The HCS separates regions of negative and positive polarities and is crucial when modelling heliospheric drifts [11, and references therein]. Another important quantity is the sign, $A$, of a Solar cycle, which is defined as $A=-1 /+1$ if the magnetic field in the northern hemisphere is negative/positive. The magnetic field in the southern hemisphere has in this picture the opposite sign. This, however, applies only to periods of low Solar activity when the magnetic field is essentially dipolar.

Figure 1 shows the magnetic field configurations, called 'magnetic field maps' in the following, obtained from GONG maps as described in [2] for several Carrington Rotations (CR) from Solar minimum $(A<0)$ in the year 2007 via Solar maximum, where the polarity changes in mid-2013 [4] and back to Solar minimum conditions $(A>0)$ in 2021. The grid resolution is $1^{\circ}$ in both longitude $(\varphi)$ and latitude $(\vartheta)$. While the sign can clearly be defined for the first and last panels, the situation in between is as unclear as is the exact determination of the time of the polarity change, i.e. between which CRs the sign of the cycle changes. In order to improve the situation we intend to generalize the quantity $A$, so that (i) it covers the entire interval from -1 to +1 and (ii) the drift reduction at high Solar activity can be self-consistently taken into account. 


\section{The new model}

We begin with a new definition of the tilt-angle based on the magnetic field maps and define a new sign, which we call the 'topological sign', $\sigma_{\mathrm{t}}$ for each CR individually, so that the change of sign can be located in time. This definition takes into account the extent of the HCS, i.e. interval $\left[90^{\circ}-\alpha_{\mathrm{N}}, 90^{\circ}+\alpha_{\mathrm{S}}\right]$, indicated by the black horizontal lines in each panel of in Figure 1.

The northern and southern tilt-angles, $\alpha_{\mathrm{N}}$ and $\alpha_{\mathrm{S}}$, are defined, as mentioned above, as the latitudical extent of the HCS and may be computed either via the distance of each point of the magnetic field map [see 4] to the HCS or by averaging the sign of the magnetic field, $q_{\mathrm{r}}$ at the heliobase, $B_{r}$, in longitude:

$$
\begin{aligned}
q_{\mathrm{r}}(\vartheta, \varphi) & =\frac{B_{\mathrm{r}}(\vartheta, \varphi)}{\left|B_{\mathrm{r}}(\vartheta, \varphi)\right|} \\
Q_{\mathrm{r}}(\vartheta) & =\left\langle q_{\mathrm{r}}(\vartheta, \varphi)\right\rangle_{\varphi}
\end{aligned}
$$

The tilt-angles are simply the first and last point in latitude when travelling from the north pole $\left(\vartheta=0^{\circ}\right)$ to the south pole $\left(\vartheta=180^{\circ}\right)$ :

$$
\begin{aligned}
& \alpha_{\mathrm{N}}=90^{\circ}-\vartheta_{\min } \\
& \alpha_{\mathrm{S}}=\vartheta_{\max }-90^{\circ} .
\end{aligned}
$$

This gives the maximum extent of the HCS over all longitudes, as can be seen in Fig. 1. Locally, i.e. as a function of longitude, this extent may be smaller, giving the values $\tilde{\vartheta}_{\min }(\varphi) \geq \vartheta_{\min }$ and $\tilde{\vartheta}_{\max }(\varphi) \leq \vartheta_{\max }$ at a given longitude, $\varphi$, which are needed for the definition of $\sigma_{\mathrm{t}}$. These angles provide the definition of an inner part $\tilde{\vartheta}_{\min }(\varphi) \leq \vartheta \leq \tilde{\vartheta}_{\max }(\varphi)$ for each longitude and an outer one, consisting of the regions $\vartheta<\tilde{\vartheta}_{\min }(\varphi)$ and $\vartheta>\widetilde{\vartheta}_{\max }(\varphi)$. If the magnetic field is dipolar with only one change of the sign of $B_{\mathrm{r}}$, the inner part collapses (first and last panel of Fig. 1), otherwise it is the latitudinal extent of the 'substructures' in the remaining panels, defined as regions with more than HCS crossing at a given longitude. Figure 2 gives the new tilt-angles (gray area and thick lines) together with the observed ones by the WSO (thin lines). The counter gives the number of longitudes with more than one HCS crossing.

There is an obvious correlation between high tilt-angles and high Solar activity, defined here as the complexity of the magnetic topology of the magnetic field maps, i.e. regions where the inner sign contributes together with the longitudinal extent of this region, visualised by the color. Moreover, this Figure shows that the newly defined tilt-angles in most CRs exceed the WSO values which are limited to $75^{\circ}$, not only during periods of high Solar activity. Note also the small period of enhanced activity in CRs 2238 and 2239, which is visible in both the WSO and newly computed tilt-angles.

\section{The topological sign}

When Solar activity is low, CR drift forms 'onion'-like loops with an orientation depending on the sign of the magnetic field. When Solar activity increases (see the middle panels of Figure 1) substructures of opposite direction might form close to the equator, resulting in inner loops with 


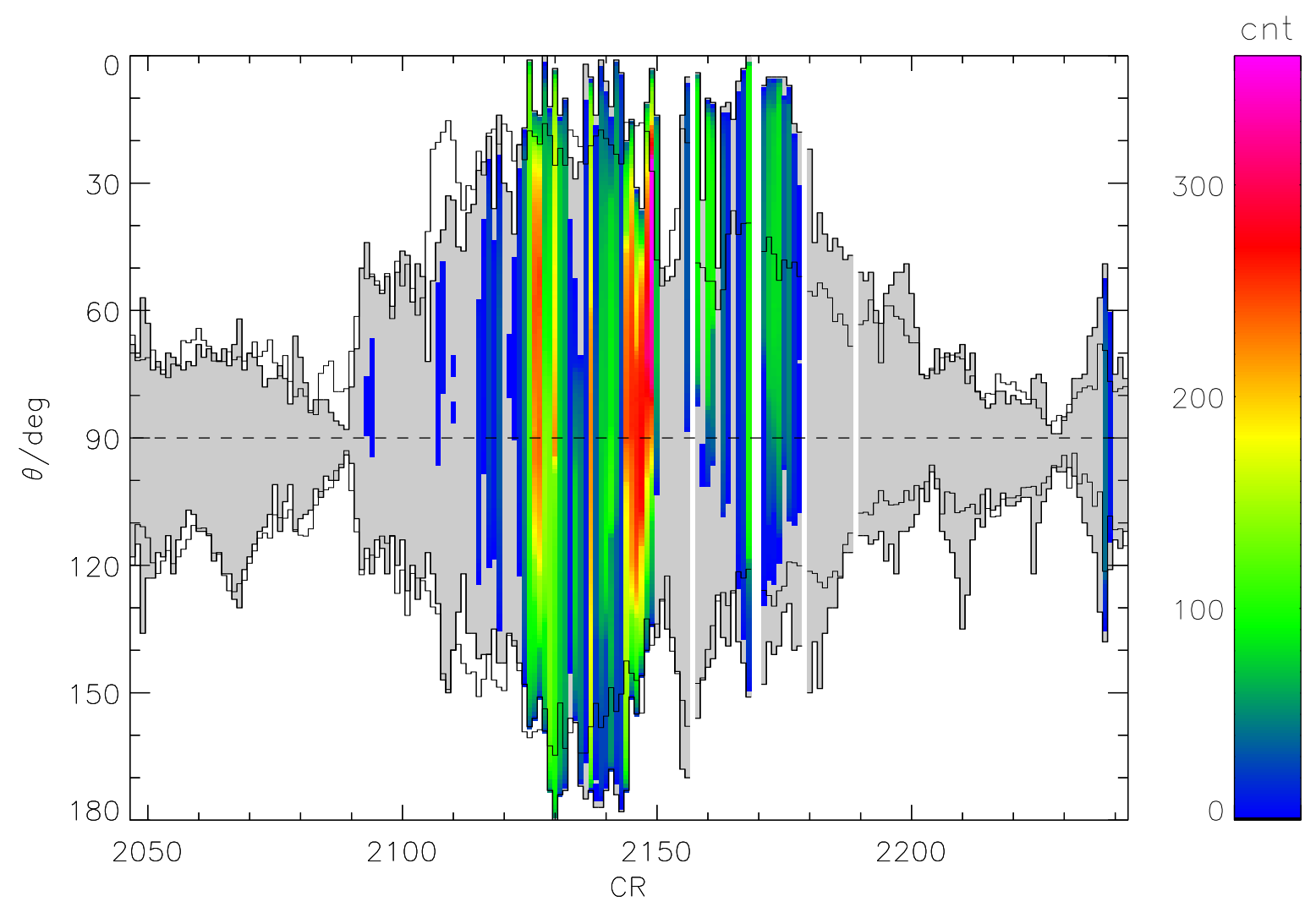

Figure 2: The newly computed (the gray area, surounded by thick lines) and WSO tilt-angles (thin lines). The color gives the number of longitudes with more than one HCS crossing. The extension of the colored bars gives the region in which these multiple HCS crossings occur, i.e. the 'inner' region.

opposite direction compared to the outer ones, and the entire drift pattern is some kind of superpostion of these two. In order to incorporate this into the definition of $\sigma_{\mathrm{t}}$, we define the signs of the inner and outer parts separately and eventually combine them into one single value for each CR.

The sign of the outer part is defined as:

$$
\sigma_{\mathrm{t}, \text { out }}(\varphi)=\frac{1}{2}\left(q_{\mathrm{r}}\left(\tilde{\vartheta}_{\min }(\varphi)-1, \varphi\right)-q_{\mathrm{r}}\left(\tilde{\vartheta}_{\max }(\varphi)+1, \varphi\right)\right)
$$

and gives the same values as the quantity $A$ above for the diopolar case.

The sign of the inner part vanishes if $\tilde{\vartheta}_{\min }(\varphi)=\tilde{\vartheta}_{\max }(\varphi)$, i.e. the HCS collapses. Otherwise, we compute the center, $\tilde{\vartheta}_{\text {cen }}(\varphi)$ between these angles and define the two averages:

$$
\begin{aligned}
& \bar{q}_{\mathrm{r}, \min }(\varphi)=\frac{\sum_{\tilde{\vartheta}_{\min }(\varphi)}^{\tilde{\vartheta}_{\mathrm{cen}}(\varphi)} q_{\mathrm{r}}(\vartheta, \varphi)}{\tilde{\vartheta}_{\operatorname{cen}}(\varphi)-\tilde{\vartheta}_{\min }(\varphi)+1} \\
& \bar{q}_{\mathrm{r}, \max }(\varphi)=\frac{\sum_{\tilde{\vartheta}_{\operatorname{cen}}(\varphi)}^{\tilde{\vartheta}_{\max }(\varphi)} q_{\mathrm{r}}(\vartheta, \varphi)}{\tilde{\vartheta}_{\max }(\varphi)-\tilde{\vartheta}_{\mathrm{cen}}(\varphi)+1} .
\end{aligned}
$$




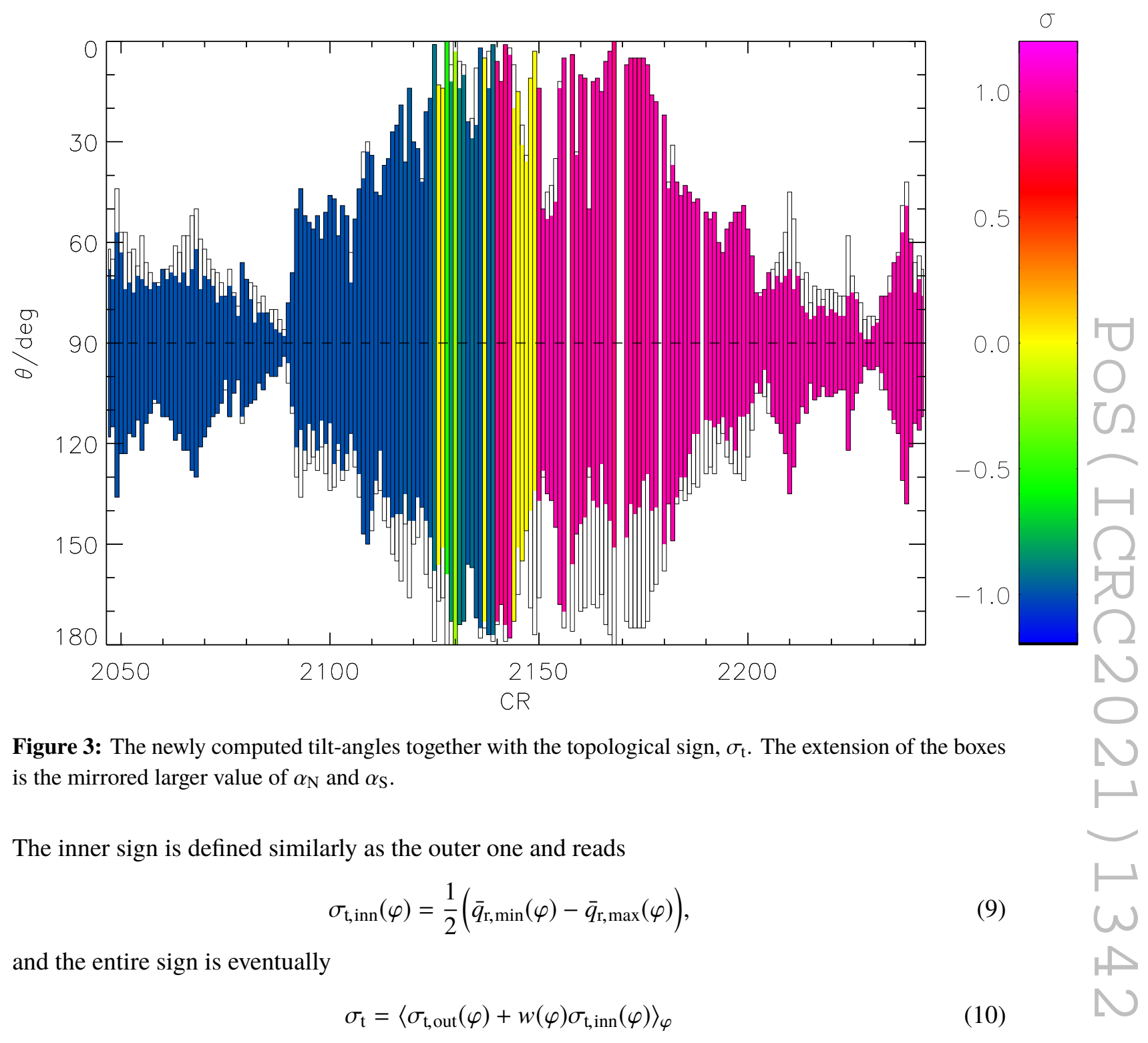

with the weight $w(\varphi)=\left(\tilde{\vartheta}_{\max }(\varphi)-\tilde{\vartheta}_{\min }(\varphi)\right) / 180^{\circ}$. The newly defined sign, $\sigma_{\mathrm{t}}$ is consistent with the previous definition via the quantity $A$, i.e. it is $-1(+1)$ during quiet times of the $A<0(A>0)$ cycle, but is able to indicate slight topological deviations, e.g. in the CRs 2161 and 2168 in Fig. 1. The polarity changes at some point between these two quiet states and is essentially zero, when the north and south poles have the same polarities (CRs 2136, 2137 or 2147, in Fig. 1). Figure 3 shows the newly computed tilt-angles $\alpha_{\mathrm{N}}$ and $\alpha_{\mathrm{S}}$ together with $\sigma_{\mathrm{t}}$, where the black boxes indicate the (mirrored) larger of the two, so that the asymmetry can be seen, in particular around CRs 2150 to 2170 , when $\alpha_{\mathrm{N}}>\alpha_{\mathrm{S}}$. As Figure 3 shows, the sign of the cycle actually changes during high Solar activity, and a closer inspection [see 4, for details] reveals that it changes between CRs 2139 and 2140 (cf. Figure 1), where $\sigma_{\mathrm{t}}$ jumps from $-1(0.928)$ to $+1(0.916)$, when the tilt-angles reach values close to $90^{\circ}$, i.e. much larger than the WSO tilt-angles which are limited to $75^{\circ}$, where observational aspects arising from the tilt of the Solar rotation axis of $7.25^{\circ}$ should be taken into account [see 4 , for details]. 


\section{Summary and conclusions}

We introduced new quantities in order to characterize the topology of the Solar magnetic field at the heliobase which is located here at 0.1AU. First, we newly defined the (northern and southern) tilt-angles as the maximum extent of the heliospheric current sheet, based on the magnetic field maps obtain from observed GONG maps. These values exceed the values obtained from the WSO, in particular during high Solar activity.

The main goal was to construct a quantity that maps the reduction of cosmic ray drifts during high Solar activity on the one hand and generalizes the quantity $A$ giving the polarity of a Solar cycle during a given Carrington Rotation on the other hand.

In order to do so, second, we defined an outer and an inner region around the HCS, where the latter region collapses when the configuration of the magnetic field maps is dipolar at a given longitude. The newly defined sign, $\sigma_{\mathrm{t}}$, consists of the outer and inner signs averaged over longitude in order to reflect the possibly complex structure of cosmic ray drift patterns.

By using this new quantity, the topological sign $\sigma_{t}$, it is now possible to model the scaling down of cosmic ray drift during periods and to self-consistently determine the sign for each Carrington Rotation during a Solar cycle. In this way it was possible to determine that the sign of the current Solar cycle occurs between CRs 2139 and 2140 in mid-2013, when the newly defined tilt-angles reach values of almost $90^{\circ}$.

\section{Acknowledgments}

This work was supported by the German Deutsche Forschungsgemeinschaft (DFG) that funded the joint project FI 706/14-3.

\section{References}

[1] M. Dumbović, B. Vršnak, J. Guo, B. Heber, K. Dissauer, F. Carcaboso et al., Evolution of Coronal Mass Ejections and the Corresponding Forbush Decreases: Modeling vs. Multi-Spacecraft Observations, Sol. Phys. 295 (2020) 104 [2006 . 02253].

[2] T. Wiengarten, J. Kleimann, H. Fichtner, P. Kühl, A. Kopp, B. Heber et al., Cosmic Ray Transport in Heliospheric Magnetic Structures. I. Modeling Background Solar Wind Using the CRONOS Magnetohydrodynamic Code, Astrophys. J. 788 (2014) 80 [1406. 0293].

[3] A. Kopp, T. Wiengarten, H. Fichtner, F. Effenberger, P. Kühl, B. Heber et al., Cosmic-Ray Transport in Heliospheric Magnetic Structures. II. Modeling Particle Transport through Corotating Interaction Regions, Astrophys. J. 837 (2017) 37.

[4] A. Kopp, J.L. Raath, H. Fichtner, M.S. Potgieter, S.E.S. Ferreira and B. Heber, Cosmic-Ray Transport in Heliospheric Magnetic Structures. III. Implications of Solar Magnetograms for the Drifts of Cosmic Rays, Astrophys. J., submitted. (2021) .

[5] J.W. Harvey, F. Hill, R.P. Hubbard, J.R. Kennedy, J.W. Leibacher, J.A. Pintar et al., The Global Oscillation Network Group (GONG) Project, Science 272 (1996) 1284. 
[6] X.P. Zhao and J.T. Hoeksema, The Magnetic Field at the Inner Boundary of the Heliosphere Around Solar Minimum, Solar Phys. 266 (2010) 379.

[7] R. Kissmann, J. Kleimann, B. Krebl and T. Wiengarten, The CRONOS Code for Astrophysical Magnetohydrodynamics, Astrophys. J. Suppl. 236 (2018) 53 [1806. 09479].

[8] A. Kopp, I. Büsching, R.D. Strauss and M.S. Potgieter, A stochastic differential equation code for multidimensional Fokker-Planck type problems, Computer Physics Communications 183 (2012) 530.

[9] M.D. Ngobeni and M.S. Potgieter, Modelling the effects of scattering parameters on particle-drift in the solar modulation of galactic cosmic rays, Adv. Space Res. 56 (2015) 1525 .

[10] J.-L. Raath, A stochastic Approach to the Modelling of Cosmic Rays in the Heliosphere, PhD thesis, North-West University, Potchefstroom, South Africa, https://repository.nwu.ac.za/handle/10394/35183 (2019) .

[11] J.L. Raath, M.S. Potgieter, R.D. Strauss and A. Kopp, The effects of magnetic field modifications on the solar modulation of cosmic rays with a SDE-based model, Adv. Space Res. 57 (2016) 1965.

[12] S.E.S. Ferreira and M.S. Potgieter, Modulation over a 22-year cosmic ray cycle: On the tilt angles of the heliospheric current sheet, Adv. Space Res. 32 (2003) 657.

[13] N.E. Engelbrecht, R.D. Strauss, J.A. le Roux and R.A. Burger, Toward a Greater Understanding of the Reduction of Drift Coefficients in the Presence of Turbulence, Astrophys. J. 841 (2017) 107.

[14] J.T. Hoeksema, J.M. Wilcox and P.H. Scherrer, The structure of the heliospheric current sheet: 1978-1982, J. Geophys. Res. 88 (1983) 9910. 